\title{
Against Division: Consciousness, Information and the Visual Streams
}

Wayne $\mathrm{Wu}$

\begin{abstract}
Milner and Goodale's influential account of the primate cortical visual streams involves a division of consciousness between them, for it is the ventral stream that has the responsibility for visual consciousness. Hence, the dorsal visual stream is a "zombie" stream. In this paper, I argue that certain information carried by the dorsal stream likely plays a central role in the egocentric spatial content of experience, especially the experience of visual spatial constancy. Thus, the dorsal stream contributes to a pervasive feature of consciousness.
\end{abstract}

\footnotetext{
I am grateful for the comments of two anonymous reviewers that led to substantial improvements in the text, to Robert Briscoe who provided helpful comments on an earlier version and to Professor Ruth Campbell for editing that improved clarity. A version of this material was presented to the Center for the Philosophy of Science at the University of Pittsburgh, to the Brain Group in the Center for the Neural Basis of Cognition at Carnegie Mellon University, and in the Neurophilosophy Forum at Georgia State University. I am grateful for the feedback I received in all those venues.
}

Address for Correspondence: Center for the Neural Basis of Cognition, 115 Mellon Institute, 4400 Fifth Avenue, Carnegie Melon University, Pittsburgh, PA 15213 Email: waynewu@cnbc.cmu.edu 
Word Count: 10,400 (inclusive notes, references, figure legends, abstract).

\section{Introduction}

Since the seminal work of Ungerleider and Mishkin (1982), the standard picture of the primate visual system identifies two separate visual streams emanating from early cortical visual areas: a dorsal stream that extends into the parietal lobe and a ventral stream that extends into the temporal lobe (see figure 1). Ungerleider and Mishkin further argued that the dorsal stream serves spatial vision ("where") while the ventral stream serves object vision (“what").

\section{Insert Figure 1}

\section{Figure redacted due to Copyright}

Figure 1: A diagram of the visual streams in the rhesus monkey. Regions V4 and MT mark the point of division of two distinct 'bottom-up' streams. V4 feeds into the ventral stream (lower shaded box), while MT feeds into the dorsal stream (upper shaded box; see section 2 on MT). Arrows indicate known anatomical feedforward and feedback connections (reprinted from Ungerleider et al. (1998), with author's permission, copyright, National Academy of Sciences, United States). Regions TEm, 45 and 12 are part of the dorsal stream (see original figure for color coding).

\section{End Figure 1}

Subsequently, Milner and Goodale (1995) advanced an influential reconceptualization of the function of the streams: the dorsal stream is for the direct control of motor action; the 
ventral stream is for perception which informs cognition. While much focus has been directed at their claims about action, I focus on their claims about consciousness. ${ }^{1}$

As we shall see, Milner and Goodale hold the following thesis of division:

Division of Consciousness: Consciousness is segregated to the ventral stream.

From this, a second thesis plausibly follows:

Division of Information: Information of relevance to consciousness is processed only by the ventral stream

It is the second thesis that helps us understand the significance of the first, so let us clarify the former. Begin with consciousness. Part of what is distinctive of consciousness is its content. The notion of content here is used in a broad sense, meant to be neutral with respect to philosophical controversies about content and consciousness. What is largely agreed upon is that we can specify the content of our visual experience by

\footnotetext{
${ }^{1}$ Recent work has argued that the ventral stream does have direct influence on action on a variety of parameters (direction of influence: ventral to dorsal). See for example (Schenk and McIntosh 2010). In a sense, our question concerns the opposite direction of influence (dorsal to ventral): does the dorsal stream contribute to consciousness and cognition? I will offer a more integrationist perspective (see final section). For some philosophical discussion with emphasis on action, see (Clark 2001) and (Briscoe 2008).
} 
describing what is experienced, such as specific objects at certain locations and of certain colors and shapes.

For neuroscientists interested in consciousness, the central idea is that conscious content is rooted in information carried by those brain areas that realize consciousness. The idea is both that changes in conscious content entail changes in neural information and that changes in realizing information entail changes in content. Like 'content', 'information' here is meant in a broad sense, namely to refer to what neuroscientists mean when they speak of the content of neural representations. ${ }^{2}$ Accordingly, as lesions in the ventral stream show, damage to visual areas carrying information about objects leads to alterations in the experience of objects (e.g. as seen in visual agnosia). Thus, Milner and Goodale $(2006,221)$ write that 'the contents of consciousness depend upon activity in the ventral stream.' Similarly, we expect that visual areas carrying spatial information will realize our spatial experience. To foreshadow my thesis, areas carrying egocentric spatial information play a role in realizing egocentric spatial visual experience. So, if consciousness is segregated to the ventral stream, then the visual information appropriately correlated with the content of consciousness is also segregated to the ventral stream. Thus, for many naturalists about the mind, the second division thesis follows from the first. Finally, a corollary of the division theses is that the dorsal stream plays no role in consciousness. This is the

\footnotetext{
${ }^{2}$ Thus, 'information' might denote Shannon mutual information, as discussed in communication theory, or Dretske's (1981) notion of indication.
} 
Zombie Thesis: The contents of the dorsal stream do not contribute to conscious vision.

By zombies, we mean the absence of phenomenal consciousness. Crick and Koch (2001) have referred to the dorsal stream as a zombie stream.

My aim in this paper is to argue against the zombie thesis. Specifically, I argue that there are informational contents in the dorsal stream that are likely candidates for realizing the egocentric spatial contents of visual experience. If so, then both theses of division are false. ${ }^{3}$ The form of my argument is as follows:

A1. Visual experience is egocentric

A2. The egocentricity of visual experience involves visual spatial constancy (including egocentric distance and egocentric representations of spatial location).

A3. Visual spatial constancy is served by the dorsal stream

A4. So: the dorsal stream serves a feature of visual experience.

Since visual spatial constancy is pervasive, instantiated with almost every movement of the eye, the following follows:

\footnotetext{
${ }^{3}$ The denial of these theses is compatible with specialization of various sorts across the two streams. It does, however, suggest a more integrationist view of the streams in respect of their contributions to normal visual experience.
} 
A5. The dorsal stream serves a pervasive feature of visual experience: visual spatial constancy.

Correlatively

A6. Some of the information inherent in the experience of spatial constancy is available to report and hence cognition.

Thus, some dorsal stream information is accessible. Finally, since spatial constancy is the constancy of visible objects and since it is uncontroversial that the ventral stream serves object recognition,

A7. The ventral stream serves visual spatial constancy.

With A5, this suggests that information from both streams must be integrated to serve consciousness in spatial constancy, hence

A8. Spatial and object information within both streams are integrated to give rise to a pervasive feature of visual experience, spatial constancy.

This suggests a more integrationist view of the dorsal and ventral streams in respect of consciousness. 


\section{Visual Consciousness and the Visual Streams}

It is uncontroversial that the ventral stream supports visual consciousness including many aspects of form and object experience. Lesions within the ventral stream often produce visual agnosia, the inability to see certain visual features including shapes and objects. ${ }^{4}$ Milner and Goodale tend to express this idea as the claim that the ventral stream is for perception, but what do they mean by 'perception'? They write: 'What we are primarily referring to is the conscious experience of seeing - that is, the visual experience we have about the current stimulus array' $(2008,775)$. They acknowledge, however, the possibility of unconscious perception. Thus, perception is not individuated by being phenomenally conscious but by its role in supporting cognition. It seems more accurate to say that the ventral stream is for cognition.

Milner and Goodale also tend to emphasize a difference in spatial information across the two streams: representations that directly guide action in the dorsal stream are egocentric or viewer-centered; those that guide cognition in the ventral stream are allocentric or object-centered. This idea is then conjoined with three additional points: (a) the action-guiding and cognition-serving representations are anatomically separated via the dorsal and ventral streams; (b) accordingly, this segregation suggests that the dorsal stream representations are not available to cognition, say for verbal report, and correlatively (c) the action-guiding representations are unconscious while the thoughtserving representations are typically conscious.

Do Milner and Goodale deny consciousness to the dorsal stream? It seems so. They write: 'conscious visual experience of the world is a product of the ventral not the

\footnotetext{
${ }^{4}$ For discussion of visual agnosia, see Farah (2004).
} 
dorsal stream' $(2004,109)$. Furthermore, '[v]isual phenomenology, in our view, can arise only from processing in the ventral stream, processing that we have linked with recognition and perception' (1995, p. 200). These points are affirmed in the appendix to the second edition (2006) of The Visual Brain in Action (see section 8.2.1 in the appendix). It is worth pointing out that they also make weaker claims: 'spatial perception, in the full sense, is associated more with the ventral stream than it is with the dorsal' (1995, p. 119, my italics). Nevertheless, the stronger claims are frequent enough, so I shall focus on them.

In response to Milner and Goodale, Ungerleider and Haxby (1994) adduced a systematic study by von Cramon and Kerkhoff (1993) examining patients with lesions in parietal cortex. These patients were tested on a variety of spatial judgment tasks that did not require their performing bodily actions. For example, in one task, subjects were asked to judge when a tilted line stood at vertical (the line, presented on a computer screen, was rotated by the experimenter until the patient judged it to be vertical). Patients with parietal lesions outside the ventral stream showed deficiencies in this task, misjudging when the line was vertically oriented. So these studies provide evidence that non-ventral stream lesions can affect conscious perception; in this case, of line orientation. ${ }^{5}$

\footnotetext{
${ }^{5}$ In respect of the discussion that follows, one patient, PU, has a lesion that seems to be localized to the superior parietal lobe and shows deficiencies in a variety of spatial tasks. For some additional cases, see (Jacob and Jeannerod 2003), chp. 3, sect. 5. Jeannerod's work often offered a strong contrastive perspective to that of Milner and Goodale (see references and discussion in Jacob and Jeannerod op. cit.).
} 
Hemi-spatial neglect is often offered as evidence that lesions outside the ventral stream affect conscious vision, namely an apparent contraction of the visual field. Neglect typically arises from damage to the right inferior parietal lobe (IPL) with subsequent neglect of the left side of space. Accordingly, neglect patients seem not to be visually aware of the left side of space, and this is often attributed to a defect in attention. Given that IPL is taken to be outside the ventral stream (IPL is sometimes referred to as carrying a 'third' stream), may not such neglect also undercut the thesis of the division of consciousness?

The crucial issue, however, is the information that is responsible for conscious content. Consider a recent review by Singh-Curry and Husain (2009) who argue that 'a primary function of the right IPL is in maintaining attention on current task goals as well as encoding salient events in the environment so that task-sets can be speedily reconfigured to deal with new challenges' (1435; they emphasize that their account of IPL is not meant on its own to explain neglect). Assume that the basis for neglect concerns a defect in attention served by regions in IPL. This finding is consistent with the thesis of the division of consciousness to the ventral stream. Milner and Goodale do not claim that the ventral stream is on its own sufficient for visual consciousness but of course depends on a proper functioning brain (2006, p. 228). In particular, attentional modulation is critical for consciousness: 'Attentional modulation of ventral-stream processing leads to conscious perception' (1995, p. 200; see also their 2006, p. 221ff). Accordingly, they can allow that defects in attention can affect consciousness. So lesions outside the ventral stream which affect consciousness by altering attention are perfectly consistent with the division of consciousness and the zombie thesis. It is the information 
carried by the ventral stream which is important for the contents of consciousness (see their appendix, 2006, for presentation of numerous examples of this). To challenge the division of consciousness, we need to identify specific informational content in the dorsal stream which contributes to consciousness.

The effects of lesions in right IPL are certainly suggestive. Milner and Goodale (1995) originally argued, however, that in humans, the dorsal stream terminates in the superior parietal lobe (SPL) and thus does not extend into IPL (the parietal lobe is divided by the intraparietal sulcus (IPS) into SPL and IPL). They acknowledge that this is contrary to most accounts which assume such lesions affect the dorsal stream (see Mattingly (1999) for discussion). In contrast, Rizzolatti and Matelli (2003) argued that the dorsal stream is divided into two distinct sub-streams: a dorso-dorsal stream that extends into SPL, and a ventro-dorsal stream that extends into IPL. ${ }^{6}$ The ventro-dorsal stream plays both a role in action and perception. Focusing on neglect resulting from lesions in IPL, Rizzolatti and Matelli conclude that 'individuals must have the parietofrontal sensorimotor circuits intact in order to achieve object perception. The ventral stream processing is not sufficient to obtain perception without parietal spatial processing' (153). ${ }^{7}$ This claim is incompatible with the theses of division only if parietal

\footnotetext{
${ }^{6}$ See also Glover's (2004) division of SPL and IPL function in terms of planning and control and the discussion in (Pisella et al. 2006).

${ }^{7}$ The original dissection of the two streams was done in work on monkeys. In those animals, the dorsal stream is understood to extend into the IPL. One criticism of Milner and Goodale's claim that the dorsal stream extends only into SPL in humans is that this suggests that the dorsal stream migrated across the intraparietal sulcus (IPS) from IPL to SPL at some point in evolutionary time.
} 
spatial processing carries information which explains correlated conscious contents. Again, Milner and Goodale need not deny that parietal spatial processing, associated with attention, is necessary for visual consciousness.

In a recent discussion, Kravitz et al. (Kravitz et al. 2011) review the divisions emanating from the dorsal stream and propose a three-fold division: a parieto-frontal pathway primarily supporting spatial working memory, a parieto-premotor pathway primarily supporting visually guided action, and a parieto-medial temporal pathway primarily supporting spatial navigation. They conclude that

“..the dorsal stream, which was originally defined as the pathway between the striate cortex and the posterior part of the inferior parietal lobule, actually comprises a widespread visuospatial processing system that contributes to both spatial perception and non-conscious spatial processing across numerous cortical areas in the frontal, temporal and limbic lobes..” (219).

The neuroanatomical issues are certainly suggestive, but what is currently lacking is specification of the precise information in dorsal areas that might play the requisite role in consciousness. This is a gap I hope to fill in what follows.

Perhaps the most promising case is visual area MT (in humans, called MT + or V5), wherein lesions affect the visual experience of motion (see (Nawrot 2003)). Neurons in MT are responsive to visual stimuli in motion, and lesions therein can lead to failure to perceive motion (akinetopsia). For example, patient LM reports that 'people were

Rizzolatti and Matelli speak of this as a 'rather strange proposal' (2003, p. 147; but see Milner's argument in his 1997). 
suddenly here or there but I have not seen them moving' (Zihl, Von Cramon, and Mai 1983, 315). That is, LM could perceive the translocation (change in spatial position), but not the intervening motion. Moreover, according to many vision theorists, MT lies squarely in the traditionally defined dorsal stream. This work on MT, of which Milner and Goodale were aware, renders the following claim of theirs puzzling: '[we] can see no convincing lesion evidence that the dorsal stream underlies visuospatial perception in either monkeys or humans (1995, p. 119).' The absence of such lesions seems to be important for their segregation of consciousness away from the dorsal stream. Yet the MT lesions provide precisely such a case. ${ }^{8}$

Milner and Goodale's original response (1995) is compressed. They point out that MT is connected with visual area V4 which lies early in the ventral stream, and note that 'such dorsal to ventral projections provide essential information for the perception and recognition both of the nature of an object or animal that is moving and of the nature of the motion itself' $(1995,51)$. Their idea must be that MT is, in some ways, like early visual areas such as V1 (primary visual cortex). Damage to V1 affects conscious vision, but only because V1 serves as a necessary relay of visual information from the eye to the visual areas serving visual consciousness and not because it is part of the machinery that

\footnotetext{
${ }^{8}$ The case of MT is surprisingly not remarked on in the literature given that most depictions of the dorsal stream include it and given the longstanding knowledge of the role of MT in motion experience though it has been independently noted by (Schenk and McIntosh 2010). Milner and Goodale (1995) seem to entertain the possibility that MT is not part of the dorsal stream (p. 202), and in a later paper, Milner (2012) does not list MT as part of the dorsal stream.
} 
directly yields conscious vision, a function of later visual areas. Thus, the ventral stream whose informational contents are correlated with conscious contents serves as the core realizer of conscious experience, explaining its specific character (Shoemaker 1987). The total realizer of consciousness, of course, includes the entire visual system.

Analogously, MT computes and relays visual motion information but is not part of the core realizer of visual consciousness. This reading is confirmed by a later discussion (Milner and Goodale 2006, 219) where Milner and Goodale suggest that given MT's connection to both streams “..it might make most sense to treat MT as an 'early' visual area, one that has the same relationship with the two streams as primary visual cortex..”. One worry is that the response begs the question. After all, Ungerleider and Mishkin's account, to which Milner and Goodale's model is a response, claims that visual experience is served by both streams: the dorsal is for spatial vision and the ventral for object vision. Ungerleider and Mishkin argued for spatial vision given the data we noted above concerning parietal lesions. From their perspective then, akinetopsia due to lesions in MT provide further confirmation that the dorsal stream (or at least parts of it) serves spatial visual experience, namely in respect of experiencing changes in spatial position inherent in motion. Milner and Goodale's perspective is that MT may be located within an early part of visual processing that projects to both streams. Clearly, the issue of MT is a controversial one.

In the rest of the paper, I assemble evidence that information carried by the dorsal stream is of crucial importance to visual spatial experience, specifically the egocentric aspects of experience that are bound up with visual spatial constancy. This argument allows us to sidestep complicated anatomical and functional issues concerning the role of 
IPL and MT vis-à-vis the dorsal stream. The informational content my argument points to likely resides in areas around IPS such as the ventral intraparietal area (VIP) and the lateral intraparietal area (LIP), two areas that all parties agree lie within the dorsal stream. Of course, the empirical issues remain open. My aim is to make a strong case that dorsal stream areas contribute to the content of consciousness so as to motivate more directed empirical work on this.

\section{Egocentric versus Allocentric Reference Frames}

Milner and Goodale tend to link egocentric representation to the dorsal stream and allocentric representation to the ventral. ${ }^{9}$ Yet, to understand this claim, we need to

\footnotetext{
${ }^{9}$ Some years ago, I presented the following argument to a well-known proponent of the Milner and Goodale model: (a) visual experience is egocentric but (b) the ventral stream only processes allocentric spatial information, so (c) the ventral stream cannot be the sole determinant of visual experience (this was the starting point of this paper). His response was to affirm (a) and deny (b). (b) is a very strong claim, and as far as I can tell, neither Milner and Goodale nor any other vision scientist has endorsed it. There is some evidence that gain-modulation is observed in neurons in the ventral stream (Lehky et al. 2008) such as eye-centered neural responses that are also modulated by head position. Gain-modulation can be used to construct body-centered representations (Zipser and Andersen 1988). Some vision scientists I have spoken to are receptive to egocentric coding in the ventral stream due to the likely failure of strict position invariance of object representations, namely the idea that an object selective neuron shows the same activity wherever the stimulus object lies in the neuron's receptive field, the region of the retina stimulation to which the neuron responds (Kravitz, Kriegeskorte, and Baker 2010). It has long been thought that object responsive neurons show position invariance. If some show position
} 
understand the notions of egocentric versus allocentric reference frames (see Klatzky (1998)). A rough outline will suffice for our purposes.

Let us begin with the standard gloss on egocentric spatial representation: it is one whose content is characterized by specifying a reference frame centered on the subject's body. I will refer to this part of the body as the reference center of the reference frame. Accordingly, when the location of a perceptible object is represented in an egocentric reference frame, the location is represented relative to the bodily reference center. Thus, visual experience is sometimes said to be eye-centered while auditory experience is said to be head-centered. In contrast, we can take allocentric representations as involving reference frames centered on objects that are not the perceiver's body. This initial division between egocentric versus allocentric reference frames, namely as a distinction between body- and non-body-centered reference frames, is sufficient for our purposes.

The standard assumption is that visual experience is egocentric. This is true, but as I shall argue in the next section, it isn't obviously true so we need an argument for it. For the moment, let us assume that visual experience is egocentric. We have seen that this implies that the spatial location of visible objects is represented relative to some part of the body. But what else is characteristic of an egocentric representation in vision? Since normal human visual experience is three-dimensional and the locations of visible variance, then these carry spatial information that are in a sense eye-centered since their activity depends on the location of the stimulus object relative to position within their receptive fields and hence relative to the eye. The relevant notion of egocentricity that I am concerned with, however, is of a reference frame that involves a more detailed three-dimensional representation of spatial information and is not necessarily eye-centered. 
objects are represented relative to the egocenter, we can present egocentric spatial content in a Cartesian coordinate system, centered on part of the perceiver, with the Cartesian axes setting egocentric directions (e.g. the z-axis defines straight ahead). More appropriate would be a spherical coordinate system that represents egocentric distance explicitly as a vector originating at the egocentric origin and whose magnitude is the distance between visible objects and the reference point.

An egocentric perceptual representation represents the location of objects relative to the perceiver and, presumably, this involves the representation of egocentric distance, the distance of objects in relation to the perceiver's body. Egocentric distance is sometimes spoken of as depth, but 'depth' is potentially ambiguous. Since our vision is binocular, let me focus on the binocular depth cue, retinal disparity (I shall pass over monocular depth cues). Consider a textbook demonstration of retinal disparity (Palmer (1999)). With your left eye closed, line up your two thumbs so that the one closest to you occludes the other. That is, they both lie on the line drawn from the fovea through the point of fixation, namely the direction of gaze. Now switch: close your right eye and open your left while fixating on the closest thumb. You will note that the thumbs are no longer superimposed, but the farther thumb looks a little to the left of the direction of gaze. Because the two eyes are themselves spatially separated, the retinal image of the thumb in one eye is spatially displaced compared with that in the other eye. The image of the thumb stimulates noncorresponding points in each retina. On the basis of this, the visual system is able to compute the depth of objects (for a recent review on disparity see (Parker 2007)). 
We must distinguish between absolute disparity, which corresponds to depth relative to the fixation point, and relative disparity, which corresponds to depth between external objects. Clearly, the absolute disparity will change as the eye moves while relative disparity will remain constant so long as external objects do not move (psychophysical evidence suggests we are more sensitive to changes in relative disparity and less so to changes in absolute disparity, Westheimer (1979)). ${ }^{10}$ The critical point is that these binocular depth cues provide allocentric depth information. They provide depth relative to another object or point in space that need not be the perceiver's body. Thus, binocular disparity does not immediately provide egocentric distance, namely depth relative to some reference point on the body.

We can compute the egocentric distance of objects from disparity, so long as we know the values of other variables. For instance, the relation between disparity, $\delta$, depth, $d$, and fixation distance, $D$, is given in the following approximate equation for separation $I$ between the eyes:

(1) $\delta \approx I^{*} \mathrm{~d} / \mathrm{D}^{2}$ (where $D>>d$ )

Where we are fixating on an object, the egocentric distance of that object is just the fixation distance. So long as we know the values of $\delta, I$, and $d$, we can calculate egocentric distance (and thus, in principle our visual system can as well). The question is where in the visual system such information is computed.

\footnotetext{
${ }^{10}$ Neri et al. (2004) provide evidence that the ventral stream processes relative disparity in a way correlated to the experience of depth.
} 


\section{Egocentric Spatial Content in Visual Experience}

Philosophical discussions of visual experience have emphasized its egocentricity (e.g. see Peacocke's (2002) discussion of scenario content or Evans (1982) discussion of perception). Thus, we are said to see things as to the left, to the right, above and behind (from here, where we are). But why should space be experienced as egocentric rather than allocentric? Two immediate replies come to mind: visual experience is egocentric (1) because egocentricity is required for visually guided action and (2) because this is obvious on introspection. Neither is sufficiently compelling in this context.

Visual egocentric content seems to be required to guide action. Nevertheless, in the context of Milner and Goodale's theory, the appeal to vision for action is problematic since the dorsal stream representations that control and guide bodily movement are held to be unconscious. We cannot then uncontroversially appeal to vision for action to establish the egocentricity of visual experience. On the second point, I am puzzled that introspection clearly suggests egocentricity. The bald claim that this is just how it seems is unconvincing. Is it clear to you, on introspection, that objects are presented to you egocentrically as opposed to allocentrically?

To press the issue, fixate on a distant object. Note that visible objects have a spatial relation to this direction of gaze. As we would naturally say (though this begs the question in the current context), some are to the left and some are to the right of my direction of gaze. Since direction of gaze is set by the orientation of the fovea relative to the external world, it might seem that this discernible structure in our experience of space is at root egocentric. But the direction of gaze is specified via two salient points: the 
fixation point and the fovea. The question then is which of these points is the reference point for visual spatial experience? Why not the fixation point? After all, it is the fixation point and not our fovea that is visible. Moreover, as we noted above, certain aspects of depth perception are tied to the fixation point. So, why shouldn't the fixation point have claim to priority? Visual experience would then be allocentric.

Fortunately, we can provide an argument for experience's being egocentric. This is provided for by the phenomenon of visual spatial constancy. To see the failure of constancy, consider that if you move the eyeball with your hand, the world seems to move (spatial inconstancy). In contrast, during normal, self-initiated eye-movements, objects do not seem to move. This is spatial constancy. Now, consider the retinal image, the projection of the world on the eye, and note that for many changes in retinal image due to movement in the world, the same changes can result from appropriate eye movement. Accordingly, the same retinal image can give rise to spatial constancy when the eye moves or spatial inconstancy when the world moves. In that sense, the retinal image is ambiguous. It underdetermines which of these two spatial experiences the perceiver is having. To resolve the ambiguity, the visual system needs more information beyond the retinal image.

Current theories of spatial constancy draw on earlier ideas of von Helmholtz (1896) that the visual system uses motor signals related to eye movement to resolve the indeterminacy of the retinal image. These hypothesized motor signals are variously called efference copies or corollary discharge. If the visual system receives a motor signal related to self-initiated eye movement, it uses this to interpret the retinal image as due to the perceiver's own movement rather than movement in the world and yields the 
experience of spatial constancy. Where there is no such motor signal, then the changes at the retina are taken to be due to the world, and we experience spatial inconstancy.

When we make a saccade, a ballistic eye movement performed on average two times per second, everything in the visual field appears to move relative to the direction of gaze since eye-movement yields a new fixation point. That is, each stable object stands in a new spatial relation to the fixation point. Thus, its retinal position relative to the fovea has changed. Eye movements thus generate massive changes in the position of objects relative to the direction of gaze and the foveal and fixation point positions that define it. Yet despite saccadic eye movement, the world remains spatially stable. How then do we represent spatial constancy?

One answer in the empirical literature emphasizes that where the world is in fact stable, all the visible objects present in the visual field before and after movement remain in constant spatial relations with each other, usually relative to some landmark like the (next or previous) target of fixation. This form of spatial constancy provides evidence to the visual system that the external world has not moved (this is sometimes called the Landmark account, (Deubel, Koch, and Bridgeman 2010)). We can call this form of spatial constancy allocentric constancy: the spatial constancy of external objects relative to each other or to a landmark. The problem is that objects can move en masse relative to the perceiver while maintaining constant spatial relations relative to each other. Alternatively, the perceiver can move relative to those objects while they maintain constant spatial relations relative to each other. In both cases, allocentric spatial constancy is consistent with two different visual experiences: spatial constancy versus 
inconstancy. So allocentric spatial constancy, like the retinal image, is not sufficient to determine whether we experience spatial constancy or inconstancy.

The problem then is this: both the retinal image and allocentric spatial constancy can be consistent with both the experience of spatial constancy and spatial inconstancy. What information is needed to disambiguate? The natural answer is egocentric spatial content. Specifically, the spatial constancy that we experience every time the eye moves is the spatial constancy of objects relative to us, say relative to our head, torso or another otherwise immobile body part. But to map the spatial constancy of objects relative to us is to deploy an egocentric spatial representation of external objects.

You might object that in the case under discussion, the external object and the relevant part of the body qua egocentric center are both immobile relative to each other, so we can raise our earlier question: why not prioritize the external object over the perceiver's body? The reason to opt for egocentricity is parsimony. Consider the movement of a visible object relative to us when we are not moving. What we visually experience is the external object moving, and in a Cartesian coordinate system, this is a change in the coordinates of the object over time relative to some reference point. Since we experience the external object and not ourselves moving, the object's change in position is plausibly referenced in relation to our body that serves as the stable origin. If we were to reverse the role of our body and object in the spatial reference frame so that the latter serves as reference point, then the experience would map us as moving relative to the external object, which is not our experience. Accordingly, this common experience of external motion is egocentrically represented. But when the object comes to a stop so that we experience it as spatially stable, we do not suddenly change reference frames so 
that the external object is now the reference point, yielding an allocentric representation against which we are mapped relative to the object. Parsimony suggests that the same egocentric reference frame is in operation throughout the experience of constancy and inconstancy in the stop and go behavior of visible objects. Hence, spatial constancy is egocentric constancy.

Spatial constancy is a pervasive feature of visual experience, since our eyes saccade about twice per second. To understand how spatial constancy is visually represented, we have found the need to invoke spatial relations relative to the perceiver's body. Such representations are egocentric, and accordingly, visual experience is egocentric. Thus, we have established premise A1.

\section{Egocentric Distance}

Given how we have argued for A1, A2 follows: visual experience involves visual spatial constancy. What then of A3?

As Milner and Goodale emphasize, it is not controversial that many regions in the dorsal stream represent space egocentrically (Colby 1998). A variety of body-centered representations have been found in the dorsal stream, especially within the posterior parietal lobe. These include representations centered on the head, limbs, and torso (see Andersen and Buneo (2002)) Moreover, these egocentric reference frames can be computed from each other. For example, from an eye-centered reference frame plus information about eye-position or movement relative to the head, say from corollary discharge, one can compute a head-centered reference frame; from a head-centered frame 
and information about position of the head relative to the torso, one can compute a torsocentered reference frame and so on.

Given A1 and the role of spatial constancy, visual experience also represents egocentric distance. Is there evidence that the dorsal stream carries this information so as to serve visual experience? I will detail two forms of evidence. First, imaging data suggests activation of dorsal stream areas during egocentric spatial tasks. Second, there is one recent patient in whom lesions in the traditionally defined dorsal stream affect judgments of egocentric distance, a result in line with long-standing observations that parietal cortex is involved in perception of distance (Holmes and Horrax 1919, 319).

Committeri et al. (2004) created a virtual environment of a castle scene where the castle served as a visual landmark. The environment included a reference object (a red ball) and two target objects (a blue and a green trashcan). Observers were virtually placed at different locations relative to these objects and were asked to judge which of two trashcans were closer either to (a) the landmark, (b) the reference object or (c) the viewer. When one of these conditions was in play information regarding the other two conditions was irrelevant. fMRI scans (BOLD oxygen levels) of observers performing this task were contrasted with a control condition requiring observers to report which trashcan was lying on its side. Activity during the egocentric judgment task resulted in defined areas of activity in the dorsal stream, leading the authors to claim that the "..[r] esults strongly demonstrate that viewer-centered (egocentric) coding is restricted to the dorsal stream and connected frontal regions, whereas a coding centered on external references requires both dorsal and ventral regions, depending on the reference being a movable object or a landmark." (1517). Consistent with this, in plotting areas of peak neural activation 
identified in 18 studies examining judgments of egocentric distance under binocular and monocular conditions, Berryhill and Olson (2009) noted activations across the occipital and parietal lobes spanning regions of the dorsal stream.

The role of the dorsal stream in coding egocentric distance is supported by neuropsychological data. In a separate study, Berryhill and Olson (2009) report a female patient, EE555, who has a lesion restricted to a region of the dorsal stream around the junction between the parietal and occipital lobes and which does not impinge on the ventral stream. The patient shows some of the features associated with Balint's syndrome, including optic ataxia as revealed by overreaching targets. As the authors report, the area lesioned in EE555 is active during processing of stereoscopic depth and likely spans visual areas $\mathrm{V} 3 \mathrm{~A}, \mathrm{~V} 7$ and region $\mathrm{hV} 4$-topo, areas traditionally localized to the dorsal stream. Moreover, while this patient shows normal visual acuity and object recognition, she also exhibits specific impairments in spatial judgments of egocentric distance. Specifically, in a task where the patient and two controls were required to judge the egocentric distance of an unfamiliar object at 6,12,24 and 36 inches, the controls reported distance with essentially perfect accuracy while EE555 consistently overestimated egocentric distance, consistent with her actual motor behavior. ${ }^{11}$

\footnotetext{
${ }^{11}$ This was seen when an unfamiliar object was used (a generic cube). When a familiar object was used (a bottle), EE555 could use this monocular distance cue to aid distance judgment, and her behavior here was no different from controls. One likely possibility is that preserved ventral stream processing can compensate for dorsal stream defects. Something similar is seen in optic ataxia where patients can compensate for defective reaches when they recognize the object (e.g. that the target is not just a cylinder but a tube of lipstick, see (Jeannerod, Decety, and Michel 1994)).
} 
Along with the MT lesions, this case seems to provide what Milner and Goodale were initially looking for: lesions in the traditionally defined dorsal stream that yield deficits in spatial cognition, in this case perceptual judgments about egocentric distance. Since egocentric distance is part of egocentric constancy, I suggest that there is initial support for A3. Hence, we can derive a version of A4: there is one feature of egocentric spatial experience supported by the dorsal stream, namely visual experience of egocentric distance. ${ }^{12}$ It is possible, however, for Milner and Goodale to respond that the areas at issue really do not belong to the dorsal stream, but serve similar functions as early visual areas. Nevertheless, we have compelling evidence that areas in the traditionally defined dorsal stream seem to contribute relevant informational contents serving consciousness and indeed cognition.

\section{Visual Spatial Constancy}

One plausible model of visual spatial constancy is of a prediction mechanism that relies on corollary discharge signals, namely motor information about impending self-initiated eye movement. The model is perhaps most simply diagrammed as follows:

\section{Figure 2 Here}

Figure redacted due to copyright; the paper from which figures 2-4 are available in this paper: http://philpapers.org/rec/WUVSC

\footnotetext{
${ }^{12}$ Robert Briscoe has pointed out to me that some of the activity and the lesion in EE555 involve IPL.
} 
Figure 2: The Transaccadic Memory Account. The '+' marks the point of fixation, with the star the visible stationary object. A saccade to the right is generated. The top ovals represent the change in retinal image over time, while the bottom identifies first the memory trace of the initial retinal image and then the prediction of the change in image given movement. This prediction draws on corollary discharge. A comparator mechanism computes the difference between the predicted and actual image (i.e., the error). Where error is high, visual stability fails and movement is experienced. Where error is low (as in this case), spatial constancy obtains. From Wu (forthcoming), with permission.

\section{End Figure}

The basic explanation is as follows. When the eyes move, there is a change in the retinal image. Correspondingly, the motor system both issues a motor command that generates the eye movement as well as a corollary discharge (or efference copy) signal carrying information about impending movement. The corollary discharge is the basis on which the visual system makes a prediction from the current retinal image to what the retinal image will be after movement. This predicted retinal image is then compared to the actual retinal image to compute the prediction error: where the prediction is accurate, there is no error; where the prediction is inaccurate, there is error. It is then claimed that where there is no error, the visual system assumes spatial constancy; where there is an error, the visual system assumes spatial inconstancy. This Transsaccadic Memory Hypothesis (Wurtz, 2011) is succinctly stated: 
“..The basic premise of the transsaccadic memory hypothesis is that visual stability is assumed unless there is specific evidence that the assumption can be rejected. In simplified outline, the transsaccadic memory proposal comprises three main stages.

First, the features of the saccadic target and of objects immediately surrounding it are stored in a transsaccadic memory. Second, after the saccade, this memory of the target and surrounding area is compared with what is now at and around the new fixation point. Finally, the outcome is evaluated. If the before-saccade and aftersaccade features are similar, the assumption of a stable visual world is met. If not, the target must have moved, and the assumption of stability fails." $(2011,496)$.

\section{A Hierarchical model of (egocentric) spatial constancy}

There is a lacuna, however, in the transsaccadic explanation. It appeals at a critical point to the assumption of spatial constancy. Unless this talk of 'assumption' is merely to be the invoking of a homunculus, what the explanation needs is to identify the relevant informational content underwriting the experience of spatial constancy. We need to show what it is for the visual system to represent objects as spatially constant. In the previous model, the output of the comparison mechanism that computes prediction error is not specified beyond registering error. An immediate problem is that error alone cannot account for the representation of spatial constancy as error signals are used in non-visual systems including the motor system to aid motor control (Wolpert and Ghahramani 2000) and in self-monitoring of mental episodes, defects of which are invoked to explain positive symptoms of schizophrenia (see my (2012) for discussion). How then is spatial constancy represented? 
I have argued that spatial constancy is an egocentric phenomenon. That is, the spatial constancy of visible objects is mapped in reference to the subject's body. Yet when the eyes move, the spatial position of visible objects relative to the eye is not stable. So, an eye-centered spatial frame alone cannot represent spatial constancy. What is needed is more egocentric spatial information. As I noted above, the dorsal visual stream maintains different body-centered spatial representations, some centered on the eye, some centered on the head, some centered on the torso or other body parts. An obvious option is to appeal to non-eye, body-centered reference frames in the dorsal stream. For example, body-centered responses have been found in area LIP (Snyder et al. 1998) and head-centered responses have been found in visual area VIP in the monkey dorsal visual stream (Duhamel, Colby, and Goldberg 1998)). How might we deploy these neural representations to explain how the visual system maintains spatial constancy in representing external objects?

Let us first consider the case where we experience spatial inconstancy in the world, namely that the world moves relative to the body. Thus:

INSERT FIGURE 3 Here

Figure redacted due to Copyright

Figure 3: (A) A subject fixates near the visual target at time $t_{1}$ and then the object moves distance $d$ to the right (direction of gaze is given by vertical arrow); (B) the object shifts in the eye-centered frame as well as in (C) the head-centered frame. Thus, the object's movement relative to fixed body position is reflected in its 
mapping in all egocentric reference frames, consistent with experienced spatial inconstancy. From $\mathrm{Wu}$ (forthcoming), with permission.

\section{End Figure 3}

For simplicity, the reference frames are given only in two-dimensions and only in headand eye-centered frames. If the visual system is to maintain accurate egocentric spatial maps, than all body-centered reference frames must update in respect of the new position of the target, as illustrated in the figure. But the upshot is that all egocentric reference frames represent a change in the object's location over time, which is to represent its having moved. Thus, the egocentric representations encode spatial inconstancy.

Consider next spatial constancy

INSERT FIGURE 4 Here Figure Redacted due to Copyright

Figure 4: (A) A subject fixates near the visual target at time $t_{1}$ and then makes a saccade to the left, with head and object in constant position (direction of gaze given by vertical arrow); (B) the object shifts in the eye-centered frame; (C) the object does not shift in the head-centered frame. Constancy in the head-centered frame in some way relies on the corollary discharge signal. The result is spatial constancy as represented in one of the egocentric frames. From $\mathrm{Wu}$ (forthcoming), with permission. 


\section{End Figure 4 Here}

Where the retinal change is due to an eye movement, the eye-centered frame registers the shift of the object's position relative to the fovea and direction of gaze, something that we visually experience. At the same time, the proposal is that the movement generates a corollary discharge signal whose function here is to cancel or compensate for any further updating of other egocentric reference frames. In effect, the visual system uses information about movement to maintain its representations. In the eye-movement case, the upshot is cancelling the updating of other egocentric frames (again, non-head centered frames are omitted). Hence, the position of the object in the head-centered frame is accurately depicted as not changing. According to the head-centered reference frame, it is as if the object has not moved relative to the head, but this is just a way of representing egocentric spatial constancy.

Whether this Hierarchical model is correct and whether it can explain a host of other constancy-related phenomena are further empirical questions. The current point is that the transsaccadic model of visual spatial constancy is silent at a crucial point, namely in specifying the relevant information that accounts for the spatial content of the experience of spatial constancy. By appealing to egocentric reference frames widely acknowledged to be present in the dorsal stream such as those in LIP and VIP, it is a fairly simple matter to fill in the lacuna. As to my knowledge, no non-eye centered egocentric representations have been definitively demonstrated in the ventral stream (e.g. head-centered representations), then if one accepts the Hierarchical model as a working hypothesis, the dorsal stream is on current evidence the most plausible neural substrate 
for the relevant egocentric representations underlying constancy. Accordingly, given the postulated deployment of different body-centered spatial reference frames in the dorsal stream, it is plausible that egocentric information in the dorsal stream serves the ubiquitous visual experience of egocentric spatial constancy.

It is worth noting that no cortical lesions have been reported to perturb spatial constancy mechanisms so that patients experience a constantly moving world with each eye movement. The lack of such patients suggests that systems supporting spatial constancy are widely distributed across multiple brain regions. Indeed, if spatial constancy can be preserved so long as one body-centered reference frame carries information that the world is constant relevant to that body part, then focal lesions are unlikely to disrupt spatial constancy and large scale lesions might have so many other effects as to mask the failure of constancy.

There is one reported neuropsychological case that I am aware of that is relevant. To understand the phenomenon at issue, stand before a bookshelf or some background with a discernible pattern. Now, raise your index finger before you and move it laterally while maintaining fixation on your fingernail. To maintain fixation, you must move your eye in a movement known as smooth pursuit. The critical feature of the experience is that while you maintain fixation on the fingernail, which you experience as moving, the background remains spatially constant. From the standpoint of the retinal image, the projection of the background onto the retina is shifting as you move your eyes. So, there must be some way that the change of retinal position of the background is compensated 
for so as to maintain the experience of constancy. ${ }^{13}$ One reported patient, RW, (Haarmeier et al. 1997) shows the following defective experience: on smooth pursuit of a moving object, the background appears to be in motion. Indeed, this induces vertigo in the patient as when he watches his children on a carousel and the background seems to move. So, the mechanism whereby the background is normally kept spatially constant during smooth pursuit movement fails. On other tests for visual functions, RW is otherwise normal. The authors note that the lesion is restricted to occipito-parietal regions, including the relevant motion areas, and they propose that the critical damage is in the human homolog of area MST in the monkey dorsal stream, a region known to be sensitive to optic flow and movement with respect to the head and which is connected to area VIP, an area with head-centered activity. ${ }^{14}$ If correct, this lesion provides another case where damage to the traditionally defined dorsal stream affects spatial vision

\footnotetext{
${ }^{13}$ We can explain the experience of constancy and inconstancy during smooth pursuit on the Hierarchical model. The objects in the background are subject to the same constancy mechanisms as noted earlier, but the object being pursued by the eye projects to a constant retinal position, namely the fovea, so relative to the eye-centered frame it is stable. But relative to a head-centered frame, it is not, and this explains why it is experienced as moving relative to the head but not the eye.

${ }^{14}$ To my knowledge, this is the only reported patient with spatial constancy defects due to cortical lesion, and the effect seems to be restricted to smooth pursuit rather than saccadic movements. Haarmeier and Kammer (2010) used transcranial magnetic stimulation (TMS) to disrupt activity in a variety of targeted brain areas. While eye movements were disrupted, spatial constancy was preserved.
} 
(though again Milner and Goodale could argue that MST is not within the dorsal stream proper).

I have proposed a plausible empirical model that implicates a role for the dorsal stream in supporting spatial constancy. Thus, I conclude that there are compelling empirical grounds for A3 in respect of spatial constancy, and thus A4: the dorsal stream serves a feature of visual experience. Since our eyes saccade two times per second, the spatial constancy of visual experience is a pervasive feature of vision, one that is present with each eye movement. Hence A5: the dorsal stream serves a pervasive feature of visual experience. Finally, the visual experience of the spatial constancy and inconstancy of objects as well as their egocentric distance are features that we can report and cognize. After all, I have relied on our access to these features in discussing the relevant experience. We have seen how damage to the dorsal stream can affect access to this information or processing of it as in patients EE555 and RW who can report to us on these features. So, A6: Some of the egocentric information inherent in experience of spatial constancy is available for report and cognition.

\section{Dorsal versus Ventral Streams, Revisited}

I have completed my argument that the dorsal stream supports a pervasive feature of the visual experience of space. Since our experience of spatial constancy is of the spatial constancy of objects and since everyone agrees that the ventral stream is critical for object vision, this suggests A7: the ventral stream serves visual spatial constancy. Given that to experience objects as spatially constant implies that we view them as egocentrically spatially constant (as per my argument for A1), then it seems that A8 is 
plausible: spatial and object information within both streams are integrated to give rise to a pervasive feature of visual experience. This means that there should be interaction between the visual streams to integrate visual information for consciousness. Milner (1997) has suggested that IPL might serve as a center for visual stream integration ${ }^{15}$ while Kravitz et al. (2011) have suggested the parieto-medial temporal stream in this regard. Future empirical work might fruitfully investigate these potential hubs for the informational integration needed for consciousness.

To summarize, I have argued that a pervasive feature of visual experience, visual spatial constancy, involves body-centered egocentric spatial content and that current evidence suggests that such egocentric information is carried in dorsal stream areas such as VIP and LIP. If so, then we have good reason to hypothesize that the dorsal stream contributes such information to the visual experience of constancy. If correct, this means that the zombie thesis, and the theses of the division of consciousness and information are incorrect. It is important to emphasize that denying these claims is consistent with holding that the two streams are specialized for different visual computations and carry different information. Indeed, it might even be true that some portions of the dorsal stream are zombie streams, carrying information that is inaccessible to consciousness and cognition (e.g. those that are tied to the direct guidance of action ${ }^{16}$ ). Nevertheless, the

\footnotetext{
${ }^{15}$ In their (2006), Milner and Goodale suggest the possibility that 'in the intact human brain, the right inferior parietal lobule (IPL) provides a flexible high-level representational medium within which the contents of visual consciousness are constructed' (228).

${ }^{16}$ But see Mole (2009).
} 
dorsal stream as a whole is not a zombie stream. My argument suggests a more integrationist perspective on a pervasive feature of visual consciousness. In respect of consciousness, the visual system might be more united than divided.

Center for the Neural Basis of Cognition Carnegie Mellon University 


\section{References}

Andersen, R. A and Buneo, C. A. 2002: Intentional maps in posterior parietal cortex. Annual Review of Neuroscience, 25, 189-220.

Berryhill, M. E., Fendrich, R. and Olson, I. R. 2009: Impaired distance perception and size constancy following bilateral occipitoparietal damage. Experimental Brain Research, 194, 381-393.

Berryhill, M. E. and Olson, I. R. 2009: The representation of object distance: evidence from neuroimaging and neuropsychology. Frontiers in Human Neuroscience 3, $1-9$.

Briscoe, R. 2008: Egocentric spatial representation in action and perception. Philosophy and Phenomenological Research, 79, 423-60.

Clark, A. 2001: Visual experience and motor action: are the bonds too tight? Philosophical Review, 110, 495-520.

Colby, C. 1998: Action-oriented spatial reference frames in cortex. Neuron, 20, 1524.

Committeri, G., Galati, G. Paradis A.-L., Pizzamiglio, L., Berthoz, A. and LeBihan, D. 2004: Reference frames for spatial cognition: different brain areas are involved in viewer-, object-, and landmark-centered judgments about object location. Journal of Cognitive Neuroscience, 16, 1517-1535.

Von Cramon, D.Y. and Kerkhoff, G. 1993: On the cerebral organization of elementary visuo-spatial perception. In B. Gulyas, D. Ottoson, and P. E. Roland (eds.), Functional Organization of the Human Visual Cortex. Oxford, Pergamon Press. 
Deubel, H., Koch, C., and Bridgeman, B. 2010: Landmarks facilitate visual space constancy across saccades and during fixation. Vision Research, 50, 249-259.

Dretske, F. 1981: Knowledge and the Flow of Information. Cambridge: MIT Press.

Duhamel, J. R., Colby, C. and Goldberg, M. E. 1998: Ventral intraparietal area of the macaque: congruent visual and somatic response properties. Journal of Neurophysiology, 79, 126-136.

Evans, G. 1982: The Varieties of Reference. Oxford: Oxford University Press.

Farah, M. J. 2004: Visual Agnosia, Second Edition. MIT Press.

Glover, S. 2004: Separate visual representations in the planning and control of action. Behavioral and Brain Sciences, 27, 3-78.

Goodale, M.A. and Milner, A.D. 2004: Sight Unseen: An Exploration of Conscious and Unconscious Vision. Oxford: Oxford University Press.

Haarmeier, T. and Kammer, T. 2010: Effect of TMS on oculomotor behavior but not perceptual stability during smooth pursuit eye movements. Cerebral Cortex, $20,2234-43$

Haarmeier, T.,Thier, P., Repnow, M., and Petersen, D. 1997: False perception of motion in a patient who cannot compensate for eye movements. Nature, 389, 849-851.

Helmholtz, H. von. 1896: Handbuch der physiologischen Optik. Hamburg: Leopold Voss.

Holmes, G. and Horrax, G. 1919: Disturbances of spatial orientation and visual attention, with loss of stereoscopic vision. Archives of Neurology and Psychiatry, 1, 385-487. 
Jacob, P. and Jeannerod, M. 2003: Ways of Seeing: The Scope and Limits of Visual Cognition. Oxford University Press.

Jeannerod, M., Decety, J. and Michel, F. 1994: Impairment of grasping movements following a bilateral posterior parietal lesion. Neuropsychologia, 32, 369-380.

Klatzky, R.L. 1998: Allocentric and egocentric spatial representations: definitions, distinctions, and interconnections. In C. Freska, C. Habel and K. F. Wender (eds.), Spatial Cognition, An Interdisciplinary Approach to Representing and Processing Spatial Knowledge. Berlin: Springer Verlag.

Koch, C. and Crick, F. 2001: The zombie within. Nature, 411, 893.

Kravitz, D. J., Kriegeskorte, N. and Baker, C. I. 2010: High-level visual object representations are constrained by position. Cerebral Cortex, 20, 2916-2925.

Kravitz, D. J., Saleem, K. S., Baker, C. I. and Mishkin, M. 201: A new neural framework for visuospatial processing. Nature Reviews. Neuroscience, 12, 217-230.

Lehky, S. R., Peng, X. McAdams, C. J., and Sereno, A. B. 2008: Spatial modulation of primate inferotemporal responses by eye position. PloS One, 3, e3492.

Mattingley, J.B. 1999: Attention, consciousness, and the damaged brain: insights from parietal neglect and extinction. Psyche, 5, 14.

Milner, A. D. and Goodale, M. A. 2008: Two visual systems re-viewed. Neuropsychologia, 46, 774-785.

Milner, A. D. 2012: Is visual processing in the dorsal stream accessible to consciousness? Proceedings of the Royal Society B: Biological Sciences, 279, 2289-2298. 
Milner, A. D. 1997: Neglect, extinction, and the cortical streams of visual processing. In P. Their and H.-O. Karnath (eds.), Parietal Lobe Contributions to Orientation in 3D Space. Heidelberg: Springer.

Milner, A.D. and Goodale, M.A. 1995: The Visual Brain in Action. Oxford: Oxford University Press.

- - - 2006. The Visual Brain in Action. 2nd ed. Oxford: Oxford University Press.

Mole, C. 2009: Illusions, demonstratives and the zombie action hypothesis. Mind, $118,995-1011$,

Nawrot, M. 2003: Disorders of motion and depth. Neurologic Clinics, 21, 609-29.

Neri, P., Bridge, H. and Heeger, D.J. 2004: Stereoscopic processing of absolute and relative disparity in human visual cortex. Journal of Neurophysiology 92, 1880-91.

Palmer, S.E. 1999: Vision Science: Photons to Phenomenology. Cambridge, MA: MIT Press.

Parker, A. J. 2007: Binocular depth perception and the cerebral cortex. Nature Reviews. Neuroscience, 8, 379-391.

Peacocke, C. 2002: Scenarios, concepts, and perception. In Y. H. Gunther (ed.), Essays on Nonconceptual Content. Cambridge, MA: MIT Press.

Pisella, L., Binkofski, F., Lasek, K., Toni, I. and Rossetti, R. 2006: No doubledissociation between optic ataxia and visual agnosia: multiple sub-streams for multiple visuo-manual integrations. Neuropsychologia, 44, 2734-2748. Rizzolatti, G. and Massimo, M. 2003: Two different streams form the dorsal visual system: anatomy and functions. Experimental Brain Research, 153, 146-157. 
Schenk, T. and McIntosh, R. D. 2010: Do we have independent visual streams for perception and action. Cognitive Neuroscience, 1, 52-78.

Shoemaker, S. 1987: Some varieties of functionalism. In Identity, Cause and Mind: Philosophical Essays. Cambridge: Cambridge University Press.

Singh-Curry, V. and Husain, M. 2009: The functional role of the inferior parietal lobe in the dorsal and ventral stream dichotomy. Neuropsychologia, 47, 14341448.

Snyder, L. H., Grieve, K. L., Brotchie, P. and Andersen, R. A. 1998:. Separate body- and world-referenced representations of visual space in parietal cortex. Nature, 394, 887-891.

Ungerleider, L. G., Courtney, S. M. and Haxby, J. V. 1998: A neural system for human visual working memory. Proceedings of the National Academy of Sciences of the United States of America, 95, 883-890.

Ungerleider, L. G. and Haxby, J. V. 1994: 'What' and 'where'in the human brain. Current Opinion in Neurobiology, 4, 157-65.

Westheimer, G. 1979: Cooperative neural processes involved in stereoscopic acuity. Experimental Brain Research, 36, 585-597.

Wolpert, D.M. and Ghahramani, Z. 2000: Computational principles of movement Neuroscience. Nat Neurosci, 3, 1212-7.

Wu, Wayne. Forthcoming. Visual spatial constancy and modularity: does intention penetrate vision? Philosophical Studies: 1-23.

—_- 2012: Explaining schizophrenia: auditory verbal hallucination and selfmonitoring. Mind and Language, 27, 86-107. 
Wurtz, R. H., Joiner, W. M. and Berman, R. A. 2011: Neuronal mechanisms for visual stability: progress and problems. Philosophical Transactions of the Royal Society B: Biological Sciences, 366, 492-503.

Zihl, J., Cramon, D. von and Mai, N. 1983: Selective disturbance of movement vision after bilateral brain damage. Brain, 106, 313-40.

Zipser, D and Andersen, R. A. 1988: A back-propagation programmed network that simulates response properties of a subset of posterior parietal neurons. Nature, 331, 679-684. 